Johnson \& Wales University ScholarsArchive@JWU

Health \& Wellness Department Faculty

Publications and Research

College of Health \& Wellness

2015

\title{
Measures of Neighborhood Quality: Self-reports of Mothers of Infant Children
}

Samantha R. Rosenthal

Johnson \& Wales University - Providence, Samantha.Rosenthal@jwu.edu

Patrick M. Vivier

Brown University

Michelle L. Rogers

Brown University

Frances M. Saadeh

Brown University

Melissa A. Clark

Brown University

Follow this and additional works at: https://scholarsarchive.jwu.edu/health_fac

Part of the Medicine and Health Sciences Commons

\section{Repository Citation}

Rosenthal, Samantha R.; Vivier, Patrick M.; Rogers, Michelle L.; Saadeh, Frances M.; and Clark, Melissa A., "Measures of Neighborhood Quality: Self-reports of Mothers of Infant Children" (2015). Health \& Wellness Department Faculty Publications and Research. 13.

https://scholarsarchive.jwu.edu/health_fac/13

This Article is brought to you for free and open access by the College of Health \& Wellness at ScholarsArchive@JWU. It has been accepted for inclusion in Health \& Wellness Department Faculty Publications and Research by an authorized administrator of ScholarsArchive@JWU. For more information, please contact jcastel@jwu.edu. 


\title{
Measures of Neighborhood Quality: Self-reports of Mothers of Infant Children
}

\author{
Samantha R. Rosenthal - Patrick M. Vivier • \\ Michelle L. Rogers • Frances M. Saadeh • \\ Melissa A. Clark
}

Published online: 4 March 2014

(c) Springer Science+Business Media New York 2014

\begin{abstract}
Poor neighborhood perceptions among mothers during pregnancy are associated with negative health outcomes such as maternal stress, preterm birth and long-term mental health problems in children. Given the increasing research on neighborhood perceptions, we examined the maternal-, child-, and neighborhood-level correlates of selfreported maternal neighborhood satisfaction at 13 months post-partum. Women were recruited from the post-partum units at Women and Infants Hospital of Rhode Island from January 5, 2009 to March 19, 2009. Unadjusted and adjusted regression coefficients were estimated using linear regression. In the full model, being a mother in excellent or very good health, being Hispanic/Latino, and having a higher or unknown household income were associated with a higher neighborhood satisfaction rating at 13 months, and having a stubborn baby, having more than a high school or GED education, as well as living in a neighborhood with a higher percent of black and Latino residents were significantly associated with a lower neighborhood satisfaction rating. Our results indicate that various aspects of the neighborhood environment, maternal characteristics, and characteristics of the child influence maternal neighborhood satisfaction postpartum. All of these characteristics should be accounted for
\end{abstract}

S. R. Rosenthal $(\bowtie) \cdot$ M. A. Clark

Department of Epidemiology, Brown University, $121 \mathrm{~S}$ Main

Street, Box G-S121, Providence, RI 02912, USA

e-mail: samantha_rosenthal@brown.edu

\section{P. M. Vivier}

Department of Health Services, Policy and Practice, Brown University, Providence, RI, USA

M. L. Rogers · F. M. Saadeh

Center for Population Health and Clinical Epidemiology, Brown

University, Providence, RI, USA when assessing the relationship between maternal neighborhood perceptions and both short- and long-term health effects in the offspring. In addition, when assessing neighborhood perception at any point in the life course, it is important to account for neighborhood- and individual-level characteristics, as well as risk factors associated with daily stress at the time of assessment.

Keywords Maternal - Neighborhood - Environment . Race/ethnicity · Baby

\section{Introduction}

Public health, medical and psychosocial literature has become increasingly interested in the effect of neighborhood conditions on individual health and well-being (Bond et al. 2012; Rocha et al. 2012; Stronegger et al. 2010; Weden et al. 2008; Colder et al. 2006; Evenson et al. 2007; Mello and Swanson 2007). Multiple studies have found that perceptions of poor neighborhood conditions are more strongly associated with poor health than objective measures of neighborhood quality (Weden et al. 2008; Wen et al. 2006; Poortinga et al. 2007). However, only a few studies have examined characteristics associated with individuals reporting poor perceptions of neighborhood quality (Latkin et al. 2009; Wen et al. 2006; Sampson and Raudenbush 2005). One study found that perceptions of more neighborhood disorder were associated with being younger, having no main romantic partner, and being depressed (Latkin et al. 2009). Another study found that lower perception of neighborhood quality was associated with loneliness, depression, hostility and stress, but not perceived social support or social networks (Wen et al. 2006). Other authors noted that neighborhood stigma based 
on the percent of black and Latino residents in communities predicted perceptions of lower neighborhood quality. Despite this recognition of neighborhood quality perceptions as an important risk factor in the literature, little investigation has been done to understand what influences perceptions of neighborhood quality at different stages in the life course.

In addition to the increase in investigation into the association between neighborhood perceptions and individual health outcomes, recent research has begun investigating the association between perinatal environmental exposures and birth outcomes as well as the long-term health of offspring. Several studies have shown that neighborhood environment during pregnancy as well as maternal stress is associated with negative birth outcomes (e.g. preterm birth, small-for-gestational-age) and poor mental health outcomes in children (e.g. attention deficit disorder, conduct disorder, and anxiety) (Auger et al. 2008; Collins et al. 1998; Glover 2011; Grizenko et al. 2012; Metcalfe et al. 2011; Vinikoor-Imler et al. 2011; Yorifuji et al. 2011). Several studies have also shown how parents' neighborhood perceptions are associated with their children's engagement in physical activity and overweight status (Weir et al. 2006; Timperio et al. 2004; Franzini et al. 2009). As more attention is paid to maternal selfreported neighborhood quality as an important risk factor, it is essential to understand what influences these reports during this stage in the life course, particularly when data are collected post-partum. Given the relationship of selfreported neighborhood quality and child health outcomes and the lack of research conducted on maternal neighborhood perceptions post-partum, our objective was to examine the correlates of self-reported maternal neighborhood perceptions at 13 months post-partum.

\section{Method}

Sample

Women were recruited from the post-partum units at Women and Infants Hospital of Rhode Island (WIHRI) from January 5, 2009 to March 19, 2009. As the seventh largest obstetrical hospital in the United States, WIHRI delivers over 8,500 babies per year, which represents $70 \%$ of Rhode Island deliveries (Care New England Health System 2011). All live births were screened for eligibility. A total of 477 mothers were excluded from the study. Multiple reasons for exclusion were possible, including: the mother was under 18 years of age $(13 \%)$; the mother was ill $(10 \%)$; the baby was stillborn or in the neonatal intensive care unit at the time of recruitment $(41 \%)$; the mother did not speak English or Spanish (10\%); residence was located outside of Rhode Island (32\%); or if they were deemed a 'family in crisis' by one of the hospital providers $(2 \%)$. Five percent were excluded for other reasons (e.g. maternal cognition issues). Participation involved completing a structured interview at the time of consent, which captured maternal and infant demographics as well as a complete residential history during pregnancy. Enrolled mothers were also invited to participate in a phone followup at 7 and 13 months post-partum. Again a complete residential history was completed along with perceptions of neighborhood quality of current residence. Mothers were given a $\$ 25$ gift card for each completed interview. A total of 740 mothers that met the eligibility criteria and provided valid responses for the variables of interest were included in the analytic sample. The study protocol was approved by the hospital and university Institutional Review Boards.

\section{Measures}

At the 13-month phone interview, mothers were asked about their satisfaction with their neighborhood, measured as follows, "There may be many things a person looks for in a neighborhood. On a scale from 1 to 10 , where a " 1 " means "not at all satisfied" and a "10" means "very satisfied", how satisfied are you with your current neighborhood?" This was the primary outcome used in the analysis.

Maternal socio-demographic characteristics were collected at the time of birth. Maternal age, insurance, and parity were obtained from hospital medical records, while race/ethnicity, household income, whether or not the mother lived with the baby's father, maternal hours of sleep received per night, having a stubborn baby, and baby and mother's health were self-reported at the 13-month interview. Mothers were also asked if they moved residences between time of birth and the 13-month interview. For each mother's residence at the time of the 13-month interview, the percentage of vacant households, the percentage of residents below poverty, the percentage of residents who were black, and the percentage of residents who were Latino by block group were determined from the 2000 United States Census (US Census Bureau 2002).

\section{Analysis}

All mothers' addresses at the 13-month interview were geocoded using Environmental Systems Research Institute ${ }^{\odot}$ ArcMap GIS software, version 9.3 (ArcMap 2008). Unmatched street addresses were manually geocoded when possible. Each geocoded residence was assigned to a United States Census block group. These data were then merged with data from the 2000 United States Census Summary File 3 (US Census Bureau 2002) to assign the neighborhood-level attributes to each geocoded residence. 
Table 1 Characteristics of Mothers

\begin{tabular}{lc}
\hline Characteristics & $\mathrm{N}=740(\%)$ \\
\hline Moved birth to 13 months & $208(28.1)$ \\
Insurance & \\
Medicaid/other/uninsured & $340(46.0)$ \\
Private & $400(54.0)$ \\
Avg. hours of sleep per night [mean (SD)] & $10(0.06)$ \\
Stubborn baby & $369(50.0)$ \\
Baby health & \\
Good/fair/poor & $97(13.1)$ \\
Excellent/very good & $643(86.9)$ \\
Maternal health & \\
Good/fair/poor & $195(26.4)$ \\
Excellent/very good & $545(73.6)$ \\
Maternal age [mean (SD)] & $29.3(0.21)$ \\
Race/ethnicity & \\
White, non-hispanic & $443(60.0)$ \\
Black, non-hispanic & $54(7.3)$ \\
Hispanic/Latino & $171(23.1)$ \\
Other & $72(9.7)$ \\
First baby & $287(39.2)$ \\
\% Vacant [mean (SD)] & $5.5(0.18)$ \\
Live with baby's father & $6.0(0.3)$ \\
Household income & $13.5(0.7)$ \\
\$30,000 & $14.2(0.5)$ \\
\$30,000-\$75,000 & $591(79.9)$ \\
More than $\$ 75,000$ & \\
Don't know/refused & $165(22.3)$ \\
Hore thation & $220(29.7)$ \\
\hline
\end{tabular}

Descriptive characteristics were calculated for all mothers in the sample. Regression coefficients were estimated for expected neighborhood satisfaction at 13 months post-partum using linear regression. After calculating unadjusted coefficients for all socio-demographic variables in Table 1, all variables were included as covariates in the final model. Table 2 shows unadjusted coefficients for all relevant characteristics as well as adjusted coefficients for the fully adjusted model. All analyses were performed using Stata, version 12 (StataCorp 2007).

\section{Results}

As shown in Table 1, the majority of mothers in the sample was white, non-Hispanic $(60.0 \%)$, had more than a high school or GED education (70.2 \%), did not move between birth and the 13-month interview (71.9\%) and had private health insurance $(54.0 \%)$. Only $39.2 \%$ of women were first-time mothers and the majority reported living with the baby's father $(79.9 \%)$. Eighty-six percent of mothers reported that their baby was in "excellent" or "very good" health, but only $73.6 \%$ reported themselves to be in "excellent" or "very good" health. Mothers lived in block groups with an average of $6 \%$ black residents, $13.5 \%$ Latino residents, $5.5 \%$ vacant housing units, and $14.2 \%$ of residents below poverty.

In bivariate analyses, having private health insurance, more sleep per night, a baby in excellent or very good health, being a mother in excellent or very good health, older, and having a higher household income were all associated with a higher neighborhood satisfaction rating at 13 months. In addition, having a stubborn baby, being black, non-Hispanic, as well as living in a neighborhood with a higher percent of vacant households, black residents, Latino residents, and residents below poverty were associated with a lower neighborhood satisfaction rating at 13 months. However, in the full model, only being a mother in excellent or very good health, being Hispanic/ Latino, and having a higher or unknown household income were associated with a higher neighborhood satisfaction rating at 13 months, and having a stubborn baby, having more than a high school or GED education, as well as living in a neighborhood with a higher percent of black and Latino residents were significantly associated with a lower neighborhood satisfaction rating.

\section{Discussion}

We found that mothers' neighborhood perceptions at 13-months post-partum were associated with neighborhood, maternal, and child characteristics. Mothers who reported having a stubborn baby were significantly less satisfied with their neighborhood. To our knowledge, no other studies have examined the impact of child characteristics on maternal neighborhood satisfaction post-partum, despite the fact that more attention is being paid to maternal neighborhood perceptions as an important risk factor for health of the offspring. Caring for an infant, whether or not a mother is doing it for the first time, can be a stressful endeavor. However, mothers who identify their baby as stubborn likely endure more daily stress because a baby's temperament is a known determinant of maternal chronic stress (Mulsow et al. 2002). This daily stress can cause negative mood and negative outlook (Bolger et al. 1989), easily lowering a mother's satisfaction with her neighborhood. 
Table 2 Expected neighborhood satisfaction among mothers at 13-months post-partum ${ }^{\text {a }}$

\begin{tabular}{|c|c|c|c|c|}
\hline & \multicolumn{2}{|c|}{ Unadjusted } & \multicolumn{2}{|c|}{ Adjusted model } \\
\hline & $\beta$ & $95 \% \mathrm{CI}$ & Adjusted $\beta$ & $95 \% \mathrm{CI}$ \\
\hline Moved birth to 13 months & -0.24 & $(-0.58,0.10)$ & -0.15 & $(-0.53,0.24)$ \\
\hline \multicolumn{5}{|l|}{ Insurance } \\
\hline Medicaid/other/uninsured & 0.00 & Ref & 0.00 & Ref \\
\hline Private & 0.37 & $(0.06,0.67)$ & -0.27 & $(-0.71,0.16)$ \\
\hline Avg. hours of sleep per night & 0.18 & $(0.09,0.27)$ & 0.08 & $(-0.02,0.18)$ \\
\hline Stubborn baby & -0.57 & $(-0.87,-0.27)$ & $-\mathbf{0 . 5 3}$ & $(-0.84,-0.23)$ \\
\hline \multicolumn{5}{|l|}{ Baby health } \\
\hline Good/fair/poor & 0.00 & Ref & 0.00 & Ref \\
\hline Excellent/very good & 0.62 & $(0.17,1.08)$ & 0.09 & $(-0.40,0.57)$ \\
\hline \multicolumn{5}{|l|}{ Maternal health } \\
\hline Good/fair/poor & 0.00 & Ref & 0.00 & Ref \\
\hline Excellent/very good & 0.73 & $(0.39,1.07)$ & 0.62 & $(0.24,0.99)$ \\
\hline Maternal age & $\mathbf{0 . 0 3}$ & $(0.01,0.05)$ & 0.00 & $(-0.03,0.04)$ \\
\hline \multicolumn{5}{|l|}{ Race/ethnicity } \\
\hline White, non-hispanic & 0.00 & Ref & 0.00 & Ref \\
\hline Black, non-hispanic & -1.00 & $(-1.60,-0.40)$ & 0.40 & $(-0.29,1.08)$ \\
\hline Hispanic/Latino & -0.34 & $(-0.71,0.04)$ & 0.90 & $(0.41,1.39)$ \\
\hline Other & -0.35 & $(-0.87,0.18)$ & 0.24 & $(-0.29,0.77)$ \\
\hline First baby & 0.18 & $(-0.13,0.50)$ & 0.14 & $(-0.20,0.49)$ \\
\hline$\%$ Vacant & -0.09 & $(-0.13,-0.06)$ & -0.03 & $(-0.07,0.01)$ \\
\hline$\%$ Black & -0.06 & $(-0.08,-0.04)$ & -0.02 & $(-0.05,-0.01)$ \\
\hline$\%$ Latino & $-\mathbf{0 . 0 3}$ & $(-0.04,-0.02)$ & $-\mathbf{0 . 0 3}$ & $(-0.04,-0.01)$ \\
\hline$\%$ Below poverty & -0.04 & $(-0.05,-0.03)$ & -0.01 & $(-0.03,0.01)$ \\
\hline Live with baby's father & 0.16 & $(-0.22,0.54)$ & -0.23 & $(-0.66,0.21)$ \\
\hline \multicolumn{5}{|l|}{ Household income } \\
\hline$<\$ 30,000$ & 0.00 & Ref & 0.00 & Ref \\
\hline$\$ 30,000-\$ 75,000$ & 0.56 & $(0.14,0.98)$ & 0.41 & $(-0.08,0.89)$ \\
\hline More than $\$ 75,000$ & 0.97 & $(0.55,1.38)$ & 0.61 & $(0.03,1.20)$ \\
\hline Don't know/refused & 0.70 & $(0.22,1.19)$ & 0.53 & $(0.02,1.04)$ \\
\hline \multicolumn{5}{|l|}{ Education } \\
\hline HS/GED or less & 0.00 & Ref & 0.00 & Ref \\
\hline More than HS/GED & 0.17 & $(-0.16,0.51)$ & -0.59 & $(-0.99,-0.20)$ \\
\hline
\end{tabular}

Bold values indicate statistical significance at an alpha threshold of 0.05

${ }^{a}$ Variables included in the adjusted model include moved birth to 13 months, insurance, average hours of sleep per night, stubborn baby, baby health, maternal health, maternal age, race/ethnicity, whether or not it was the mother's first baby, household income, maternal education, whether or not the mother lives with baby's father, and block group characteristics such as \% vacant households, \% below poverty, \% Latino, and $\%$ Black

When considering neighborhood characteristics, unlike other studies (Ross and Mirowsky 2001; Skogan 1990; Wen et al. 2006; Weden et al. 2008), we did not find that objective measures of neighborhood socioeconomic status (SES) such as the percent of vacant households and residents below poverty were significantly associated with maternal neighborhood perceptions. Rather, these economic indicators are likely correlated with socio demographic characteristics that are more strongly associated with neighborhood perceptions that were significant in the model such as the percent of minority residents in the neighborhood. It is also likely that the relationship between objective neighborhood economic indicators and a mother's perception of her neighborhood was mediated by the daily stress associated with living in a neighborhood, which we accounted for with maternal and child characteristics such as self-rated health and having a stubborn baby.

Neighborhood characteristics that were associated with maternal neighborhood satisfaction included the percent of 
black and Latino residents in the neighborhood. This is consistent with Sampson and Raudenbush's (2005) findings that Americans have persistent beliefs linking the presence of ethnic/racial minority groups to qualities that are undesirable in neighbors or neighborhoods and that this phenomenon exists despite the race/ethnicity of the observer. We found that the percent minority population in a mother's neighborhood was significantly associated with lower neighborhood satisfaction even when controlling for that mother's own race/ethnicity.

Several maternal characteristics were also associated with maternal neighborhood satisfaction at 13 months postpartum. Having more than a high school or GED education was associated with lower maternal neighborhood satisfaction. It is likely that, when controlling for other socioeconomic variables, being more educated may be linked with mothers having higher expectations of their neighborhood environments or being more critical of their neighborhoods. On the other hand, having a high or unknown income, being Hispanic/Latina, and being in excellent/very good health were all maternal characteristics associated with higher neighborhood satisfaction. The association between higher household income and higher neighborhood satisfaction is not novel. Several studies have shown that, even when controlling for neighborhood SES and self-reported health status, individual-level SES is associated with neighborhood perception (Wen et al. 2006; Poortinga et al. 2007). It cannot be determined, however, if individual-level SES is a mediator in the relationship between neighborhood SES and neighborhood perception or a confounder of that relationship. Clearly household income or means would likely impact the ability to live in a neighborhood of a mother's choosing, or to her satisfaction. Being a Hispanic/Latina mother was associated with higher neighborhood satisfaction in the full model, yet this same association did not exist in bivariate analysis. Particularly, when controlling for the percent of Latino residents in the neighborhood, Hispanic/Latina mothers had significantly higher neighborhood satisfaction. This is likely due to the fact that Hispanic/Latina mothers derive more social support from and find more social cohesion in neighborhoods with higher concentrations of Hispanic/Latino residents, unlike mothers of other racial/ethnic backgrounds (Mulvaney-Day et al. 2007; Rios et al. 2011). Mothers' reports of being in very good or excellent health were also associated with significantly higher neighborhood satisfaction. Again, this is not a novel finding. Rather, several studies have shown an association between individual's neighborhood perceptions and self-rated health (Wen et al. 2006; Latkin et al. 2009; Poortinga et al. 2007). Self-rated health likely acts as a proxy for daily stress, a characteristic also shown to be related to neighborhood perceptions (Wen et al. 2006); and psychological stress has been shown to increase one's susceptibility to sickness (Cohen et al. 1993).

There are several limitations of this study. First, there may be residual confounding in the final model due to confounding by unmeasured variables or due to the collinearity that likely exists between maternal and neighborhood characteristics. Also, although we were able to account for whether or not mothers moved between birth and the 13-month interview, we have not accounted for the reasons for moving, which likely would also influence a mother's satisfaction with her neighborhood. Finally, neighborhood characteristics were pulled from the 2000 United States' census, which may have changed by the time of the 13-month interview.

As more attention is paid to maternal self-reported neighborhood quality as an important risk factor, it is essential we understand what influences these reports at this stage in the life course, particularly when data are collected post-partum. Our results indicate that various aspects of the neighborhood environment, maternal characteristics, and characteristics of the child influence maternal neighborhood satisfaction post-partum. All of these characteristics should be accounted for when assessing the relationship between maternal neighborhood perceptions and both short- and long-term health effects in the offspring. In addition, when assessing neighborhood perception at any point in the life course, it is important to account for neighborhood- and individual-level characteristics, as well as risk factors associated with daily stress at the time of assessment.

\section{References}

ArcMap (2008). (Version 9.3). Redlands, CA: Environmental Systems Research Institute.

Auger, N., Daniel, M., Platt, R., Wu, Y., Luo, Z., \& Choiniere, R. (2008). Association between perceived security of the neighborhood and small-for-gestational-age birth. Pediatric and Perinatal Epidemiology, 22, 467-477.

Bolger, N., DeLongis, A., Kessler, R. C., \& Schilling, E. A. (1989). Effects of daily stress on negative mood. Journal of Personality and Social Psychology, 57, 808-818.

Bond, L., Kearns, A., Mason, P., Tannahill, C., Egan, M., \& Whitley, E. (2012). Exploring the relationships between housing, neighbourhoods and mental wellbeing for residents of deprived areas. BMC Public Health, 12, 48.

Care New England Health System (2011). About women and infants hospital. http://www.womenandinfants.org/body.cfm?id=89\&action= detail\&ref=469. 11 April 2011.

Cohen, S., Tyrrell, D. A., \& Smith, A. P. (1993). Negative life events, perceived stress, negative affect, and susceptibility to the common cold. Journal of Personality and Social Psychology, 64, 131-140.

Colder, C., Lengua, L., Fite, P., Mott, J., \& Bush, N. (2006). Temperament in context: Infant temperament moderates the relationship between perceived neighborhood quality and behavior problems. Journal of Applied Developmental Psychology, 27, 456-467. 
Collins, J., David, R., Symons, R., Handler, A., Wall, S., \& Andes, S. (1998). African-American mothers' perception of their residential environment, stressful life events, and very low birthweight. Epidemiology, 9, 286-289.

Evenson, K., Scott, M., Cohen, D., \& Voorhees, C. (2007). Girls' perception of neighborhood factors on physical activity, sedentary behavior, and BMI. Obesity, 15, 430-445.

Franzini, L., Elliott, M., Cuccaro, P., Schuster, M., Gilliland, J., Grunbaum, J., et al. (2009). Influences of physical and social neighborhood environment on children's physical activity and obesity. American Journal of Public Health, 99, 271-278.

Glover, V. (2011). Annual research review: Prenatal stress and the origins of psychopathology: An evolutionary perspective. Journal of Child Psychology and Psychiatry and Allied Disciplines, 52, 356-367.

Grizenko, N., Fortier, M., Zadorozny, C., Thakur, G., Schmitz, N., Duval, R., et al. (2012). Maternal stress during pregnancy, ADHD symptomatology in children and genotype: Gene-environment interaction. Journal of Canadian Academy of Child and Adolescent Psychiatry, 21, 9-15.

Latkin, C., German, D., Hua, W., \& Curry, A. (2009). Individual-level influences on perceptions of neighborhood disorder: A multilevel analysis. Journal of Community Psychology, 37, 122-133.

Mello, Z., \& Swanson, D. (2007). Gender differences in African American adolescents' personal, educational, and occupational expectations and perceptions of neighborhood quality. Journal of Black Psychology, 33, 150-168.

Metcalfe, A., Lail, P., Ghali, W., \& Sauve, R. (2011). The association between neighborhoods and adverse birth outcomes: A systematic review and meta-analysis of multi-level studies. Pediatric and Perinatal Epidemiology, 25, 236-245.

Mulsow, M., Caldera, Y. M., Pursley, M., Reifman, A., \& Huston, A. C. (2002). Multilevel factors influencing maternal stress during the first three years. Journal of Marriage and Family, 64, 944-956.

Mulvaney-Day, N. E., Alegria, M., \& Sribney, W. (2007). Social cohesion, social support, and health among Latinos in the United States. Social Science and Medicine, 64, 477-495.

Poortinga, W., Dunstan, F. D., \& Fone, D. L. (2007). Perceptions of the neighbourhood environment and self rated health: A multilevel analysis of the caerphilly health and social needs study. BMC Public Health, 7, 285.

Rios, R., Aiken, L. S., \& Zautra, A. J. (2011). Neighborhood contexts and the mediating role of neighborhood social cohesion on health and psychological distress among hispanic and nonhispanic residents. Annals of Behavioral Medicine, 43, 50-61.

Rocha, K., Perez, K., Rodriguez-Sanz, M., Obiols, J., \& Borrell, C. (2012). Perception of environmental problems and common mental disorders (CMD). Social Psychiatry and Psychiatric Epidemiology (In press).

Ross, C. E., \& Mirowsky, J. (2001). Neighborhood disadvantage, disorder, and health. Journal of Health and Social Behavior, 42, 258-276.

Sampson, R. J., \& Raudenbush, S. W. (2005). Neighborhood stigma and the perception of disorder. Focus, 24, 7-11.

Skogan, W. (1990). Disorder and decline: Crime and the spiral of decay in American neighborhoods. New York, NY: Free Press.

StataCorp. (2007). Stata statistical software: Release 10. College Station, TX: StataCorp LP.

Stronegger, W., Titze, S., \& Oja, P. (2010). Perceived characteristics of the neighborhood and its association with physical activity behavior and self-rated health. Health and Place, 16, 736-743.

Timperio, A., Crawford, D., Telford, A., \& Salmon, J. (2004). Perceptions about the local neighborhood and walking and cycling among children. Preventive Medicine, 38, 39-47.

US Census Bureau (2002). Summary file 3: 2000 census of population and housing. Rhode Island.

Vinikoor-Imler, L., Messer, L., Evenson, K., \& Laraia, B. (2011). Neighborhood conditions are associated with maternal health behaviors and pregnancy outcomes. Social Science and Medicine, 73, 1302-1311.

Weden, M., Carpiano, R., \& Robert, S. (2008). Subjective and objective neighborhood characteristics and adult health. Social Science and Medicine, 66, 1256-1270.

Weir, L., Etelson, D., \& Brand, D. (2006). Parents' perceptions of neighborhood safety and children's physical activity. Preventive Medicine, 43, 212-217.

Wen, M., Hawkley, L., \& Cacioppo, J. (2006). Objective and perceived neighborhood environment, individual SES and psychosocial factors, and self-rated health: an analysis of older adults in Cook County, Illinois. Social Science and Medicine, 63, 2575-2590.

Yorifuji, T., Naruse, K., Kashima, S., Ohki, S., Murakoshi, T., Takao, S., et al. (2011). Residential proximity to major roads and preterm births. Epidemiology, 22, 74-80. 